\title{
Visual Hallucinations in a Patient with Horner's Syndrome Secondary to Internal Carotid Dissection
}

\author{
Amardeep Singh Panteleimon Mortzos Torben Lykke Sørensen \\ Clinical Eye Research Unit, Department of Ophthalmology, Copenhagen University \\ Hospital Roskilde, Roskilde, Denmark
}

\section{Key Words}

Horner's syndrome · Visual hallucinations · Carotid artery dissection · Peduncular hallucinosis · Cerebral ischemia

\begin{abstract}
A 67-year-old female presented with post-ganglionic Horner's syndrome. In addition to the classical symptoms of Horner's syndrome, the patient reported experiencing frightening complex visual and auditory hallucinations on two different occasions. Magnetic resonance angiography of the cerebrum, neck and upper thorax revealed internal carotid dissection. The symptoms and hallucinatory experiences resolved soon after antiplatelet therapy was commenced. We propose peduncular hallucinosis as the underlying mechanism.
\end{abstract}

(C) 2014 S. Karger AG, Basel

\section{Introduction}

Horner's syndrome (HS) is characterized by ipsilateral upper lid ptosis with mild elevation of the lower lid, ipsilateral mild miosis and occasionally ipsilateral conjunctival injection, apparent enophthalmos, ipsilateral facial anhydrosis and dysgeusia [1]. The cause of HS is disruption of the oculosympathetic nerve supply anywhere from its origin in the dorsolateral hypothalamus to the eye. The lesion may be central if it affects the first-order neuron as it travels from the dorsolateral hypothalamus down the brainstem to the ciliospinal center of Budge at the level of C8-T2; pre-ganglionic if it affects the second-order neuron as it passes from the ciliospinal center to the superior cervical ganglion in the neck, or post-ganglionic if the third neuron is affected as it ascends along the internal carotid

Amardeep Singh

Department of Ophthalmology

Copenhagen University Hospital Roskilde, Køgevej 7-13

DK-4000 Roskilde (Denmark)

E-Mail a.singh.opht @gmail.com 
Singh et al.: Visual Hallucinations in a Patient with Horner's Syndrome Secondary to Internal Carotid Dissection

artery to enter the cavernous sinus where it joins the ophthalmic division of the trigeminal nerve.

Carotid artery dissection (CAD) is a potentially life-threatening cause of post-ganglionic HS and may occur spontaneously or after blunt trauma. The incidence of spontaneous CAD is $2.5-3$ per 100,000 and $1-3 \%$ after blunt trauma $[2,3]$. Since the risk of stroke and permanent neurologic deficits is increased in CAD, it is essential to identify this rare condition and initiate relevant antithrombotic therapy as soon as possible [1]. Typically, the patient presents with headache, amaurosis fugax, neck swelling, pulsatile tinnitus, vertigo or scintillating scotomas (similar to those that occur in migraine) [4-6]. We report a patient presenting with complex and vivid visual hallucinations (VHs) in addition to the classic symptoms of post-ganglionic HS.

\section{Case Report}

A 67-year-old female presented with a 3-week history of persistent right-side neck pain and headache, intermittent vertigo, mild dysgeusia and complex VHs on 2 different occasions. The first experience occurred in the afternoon in a well-lit bedroom. An image of a man wearing a red and black shirt was seen by the patient sitting at her bedside and staring at her in silence. Frightened and believing she had witnessed a ghost, she screamed out loud and attempted to push the image away, but her arms would move through it. The second experience came about $2 \frac{1}{2}$ weeks later and occurred at night as the patient was about to go to sleep. The patient reported seeing an image of a man wearing a black jacket and flat cap knocking at her window from outside. The patient had insight into the untrue nature of this experience, particularly as she resided on a higher floor where it would be practically impossible to stand outside the window. Interestingly, the patient was able to hear the knocking sound. The knocking stopped as soon as the patient stood up from her bed. Both experiences lasted approximately a minute each and were associated with an acute attack of severe headache.

The patient's medical history included vitiligo. There was no history of migraine, epilepsy or other neuropsychiatric conditions. The patient denied any previous head or body trauma. She was not a substance abuser, although she did admit to taking a tablet of morphine prior to her second hallucination. Apart from oral magnesium for leg cramps and Priorin (herbal hair-strengthening product consisting of vitamins $\mathrm{B}_{5}$ and $\mathrm{B}_{7}$, cystine and extract of millet), she did not take any medications on a regular basis. On examination, bestcorrected visual acuity was 1.0 in the right eye and 0.6 in the left eye (known amblyopia), and ocular tension was measured at 19/18 $\mathrm{mm} \mathrm{Hg}$. A ptosis of the upper eyelid and relative miosis with preserved ability to constrict on light was observed on the right side. No facial anhydrosis was noted. Motility was normal, and there was no nystagmus. Slit-lamp examination was unremarkable apart from mild conjunctival injection in both eyes. The cocaine test (cocaine 10\%) was positive for HS, so a magnetic resonance angiography (MRA) scan of the cerebrum, neck and upper thorax was requested. The radiologist described a thickened internal right carotid artery near the cranial base just before the entry into the carotid channel; this was compatible with a right-sided carotid dissection (fig. 1). No signs of hemorrhage, infarction or space-occupying lesions were found. The patient was then referred to the neurovascular unit at our institution, and shortly after starting oral antiplatelet therapy with clopidogrel $75 \mathrm{mg}$ once daily, her symptoms (right-side neck pain and headache, intermittent vertigo, mild dysgeusia, and complex VHs) improved. 
Singh et al.: Visual Hallucinations in a Patient with Horner's Syndrome Secondary to Internal Carotid Dissection

\section{Discussion}

In this case report, we present a patient who experienced complex VHs in addition to other symptoms following unilateral HS secondary to carotid dissection. The clinical presentation is attributed to carotid dissection at the level of the third-order neuron. The proposed mechanism behind dysgeusia in CAD is thought to be compression of cranial nerve IX against the expanding wall of the dissecting artery [7]. Partial facial anhydrosis is absent in third-order neuron HS as the sudomotor and vasomotor fibers to most of the face separate at the superior cervical ganglion. VHs have not previously been described in patients with carotid dissection or HS; thus, the presence of VHs in this patient was particularly interesting.

VHs are defined as the perception of an object or event in the absence of an external stimulus. These may be complex where the content consists of formed, vivid and wellorganized images, or elementary where the individual experiences photopsias or shadows. The underlying mechanisms in VHs are poorly understood, although Manford and Andermann [8] review 3 pathogenic mechanisms which may underlie VH: (1) irritative processes in the association cortex generating discharges falsely interpreted as due to sensory inputs; (2) release phenomena due to defective visual input causing faulty cortical stimulation, and (3) faulty visual processing in which inputs are normal but lesions result in an inappropriate pattern of cortical excitation. Sometimes considered a manifestation of psychiatric pathology, VHs also occur in the setting of neurological or ophthalmological conditions.

In ophthalmology, the Charles Bonnet syndrome represents an important and common cause of complex VHs in patients with partial or complete visual loss. This is believed to result from lack of visual stimuli to the cortical centers leading to complex VHs with preserved insight into the untrue nature of the hallucinatory experiences [9]. Another wellrecognized cause of VHs is migraine, which causes the patient to see flickering and zigzag lines [6]. Complex hallucinations may also occur in the recovery phase of the headache and are associated with familial hemiplegic migraine [10]. It is unlikely that the VHs observed by our patient were attributable to either the Charles Bonnet syndrome or migraine as she had relatively good vision and no history of migraine.

Studies of cerebral perfusion during hallucinations demonstrate that the lateral temporal cortex, corpus striatum, and thalamus are the regions that are most likely to be responsible for the genesis of the hallucinatory experiences [11]. Peduncular hallucinosis results from vascular or infective lesions of the midbrain or adjacent structures and is a cause of VHs that often develop within a few days of the initial insult and are thought to be a release phenomenon involving colorful, vivid scenes with people, animals and other patterns $[12,13]$. They may occasionally accompany auditory hallucinations, which may explain why our patient could hear the image knocking on her bedroom window [8]. Patients cope well with their experiences once they develop insight into the unreal nature of their hallucinations, without features of paranoia or psychiatric disturbance, as was the case in our patient [14]. Thus, a possible cause of VHs in our patient is peduncular ischemia caused by CAD [5]. Although peduncular hallucinosis is most commonly associated with midbrain pathology, we did not observe this on the MRA scan. However, when localized or focal hypoperfusion of the cerebrum occurs due to transient ischemic attacks following internal carotid artery stenosis or embolism, injury to the midbrain is not always evident upon imaging [15]. In fact, another case of peduncular hallucinosis as a manifestation of a transient ischemic attack syndrome is documented, where magnetic resonance imaging and electroencephalogram revealed no abnormalities [15]. 
Singh et al.: Visual Hallucinations in a Patient with Horner's Syndrome Secondary to Internal Carotid Dissection

In conclusion, we present a patient with post-ganglionic HS due to an internal CAD with complex VHs. The hallucinations ceased after treatment for CAD was initiated. It is not entirely clear how CAD could lead to VHs. We propose peduncular hallucinosis as the underlying mechanism.

\section{References}

1 Walton KA, Buono LM: Horner syndrome. Curr Opin Ophthalmol 2003;14:357-363.

$\checkmark 2$ Schievink WI: Spontaneous dissection of the carotid and vertebral arteries. N Engl J Med 2001;344:898-906.

-3 Baker WE, Wassermann J: Unsuspected vascular trauma: blunt arterial injuries. Emerg Med Clin North Am 2004;22:1081-1098.

4 Demiryoguran NS, Karcioglu 0, Topacoglu H, Aksakalli S: Painless aortic dissection with bilateral carotid involvement presenting with vertigo as the chief complaint. Emerg Med J 2006;23:e15.

-5 Baumgartner RW, Bogousslavsky J: Clinical manifestations of carotid dissection. Front Neurol Neurosci $2005 ; 20: 70-76$

6 Wilkinson F: Auras and other hallucinations: windows on the visual brain. Prog Brain Res 2004;144:305320 .

7 Taillibert S, Bazin B, Pierrot-Deseilligny C: Dysgeusia resulting from internal carotid dissection. A limited glossopharyngeal nerve palsy. J Neurol Neurosurg Psychiatry 1998;64:691-692.

$>8$ Manford M, Andermann F: Complex visual hallucinations. Clinical and neurobiological insights. Brain 1998;121:1819-1840.

9 Menon GJ, Rahman I, Menon SJ, Dutton GN: Complex visual hallucinations in the visually impaired: the Charles Bonnet Syndrome 2003;48:58-72.

10 Ophoff RA, Terwindt GM, Vergouwe MN, van Eijk R, Oefner PJ, Hoffman SM, Lamerdin JE, Mohrenweiser HW Bulman DE, Ferrari M, Haan J, Lindhout D, van Ommen GJ, Hofker MH, Ferrari MD, Frants RR: Familial hemiplegic migraine and episodic ataxia type- 2 are caused by mutations in the $\mathrm{Ca}^{2+}$ channel gene CACNL1A4. Cell 1996;87:543-552.

$\checkmark 11$ Adachi N, Watanabe T, Matsuda H, Onuma T: Hyperperfusion in the lateral temporal cortex, the striatum and the thalamus during complex visual hallucinations: single photon emission computed tomography findings in patients with Charles Bonnet syndrome. Psychiatry Clin Neurosci 2000;54:157-162.

-12 Kumar R, Behari S, Wahi J, Banerji D, Sharma K: Peduncular hallucinosis: an unusual sequel to surgical intervention in the suprasellar region. Br J Neurosurg 1999;13:500-503.

13 Goodwin DW, Rosenthal R: Clinical significance of hallucinations in psychiatric disorders. A study of 116 hallucinatory patients. Arch Gen Psychiatry 1971;24:76-80.

14 Baska RE: Hypothalamic-midbrain dysregulation syndrome. J Child Neurol 1992;7:116-117.

15 Wenzel C, Pearlstein L: Peduncular hallucinosis as a transient ischemic attack. J Am Osteopath Assoc 1993;93:129-130. 
Case Reports in

Ophthalmology
Case Rep Ophthalmol 2014;5:347-351

DOI: $10.1159 / 000368332$

Singh et al. Visual Hallucinat

Internal Carotid Dissection

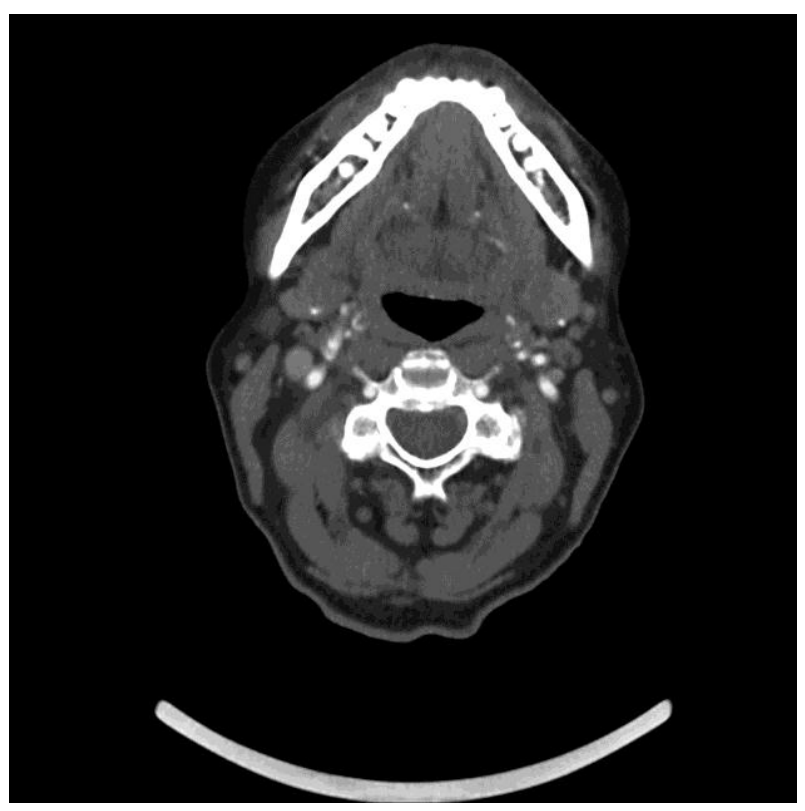

Fig. 1. Magnetic resonance image of the patient showing a thickened right internal carotid artery with a narrow lumen compatible with a diagnosis of carotid dissection. No signs of hemorrhage, infarction or space-occupying lesions were observed. 